\title{
Interference Power Characterization in Directional Networks and Full-Duplex Systems
}

\author{
Ayman T. Abusabah ${ }^{1,2}$, Rodolfo Oliveira ${ }^{1,2}$, and Luis Irio ${ }^{2}$ \\ ${ }^{1}$ Departamento de Engenharia Electrótecnica e de Computadores, Faculdade de \\ Ciências e Tecnologia, Universidade Nova de Lisboa, Portugal \\ ${ }^{2}$ Instituto de Telecomunicações, Portugal
}

\begin{abstract}
This paper characterizes the aggregate interference power considering both directional millimeter-wave (mmWave) and In-Band Full-Duplex (IBFDX) communications. The considered scenario admits random locations of the interferers. The analysis considers a general distance-based path loss with a sectored antenna model. The interference caused to a single node also takes into account the residual self-interference due to IBFDX operation. The main contribution of the paper is the characterization of the interference caused by both transmitting nodes and full-duplex operation for different parameters and scenarios.
\end{abstract}

Keywords: In-Band Full-Duplex communications, Directional Beamforming, Millimeter-Wave, Interference Characterization, Performance Analysis.

\section{Introduction}

In-Band Full-Duplex (IBFDX) communications, the nodes can transmit and receiving the signals simultaneously on the same frequency band. Compared with half-duplex communication systems, e.g., time division duplexing (TTD) and frequency division duplexing (FDD), the full-duplex systems can potentially double the capacity of the communication link, by reducing the amount of residual self-interference (SI) through passive and active methods $[1,2]$. On the other hand, directional communication is a promising technology that has an advantage in overcoming the high isotropic path loss at high-frequency bands, e.g., millimeter-wave (mmWave) band, [3]. Rigorously speaking, directional communication networks will change the conventional concepts about the interference, i.e., as the nodes only focus their beams toward a specific directed spatial channel.

The characterization of interference in wireless networks has been a topic of extensive research in the last years [4]. The aggregate interference is usually modeled through advanced stochastic geometry techniques [5] that have into account the spatial position of each interferer and its radio channel to determine the amount of interference caused to a specific node [4]. In [6], the authors have adopted the concepts of aligned gain and misaligned gain to derive a coverage model for the signal-to-interference-plusnoise ratio (SINR). The spatial interference caused by multiple simultaneous and uncoordinated transmissions occurring in the mmWave band was studied in [7] to characterize the collision probability as a function of the antenna patterns and the 
density of the transmitting nodes. The authors in [8] have also considered the effect of non-binary object blockage in the aggregate interference, by assuming that a single obstacle can cause a partial blockage. The modeling of interference in cellular MIMO beamforming mmWave communications was also tackled in [9], by considering two models (inverse Gaussian and the inverse Weibull) and a mixture of them. On the other hand, the statistical characterization of residual SI has received limited attention due to the difficulty of the mathematical modeling process. The amount of cancellation and the strength of the residual SI were computed in [10]. The authors adopted a narrowband signal model to characterize the residual SI power, i.e., it is assumed that the signal time is less than the coherence time of the channel. The similarity of the residual SI distribution with known distributions was analyzed [11], and a closed-form approximation was presented in [12] and [13]. The estimation of the channel with a single tap delay was studied in [14]. While the aforementioned works deal with each type of interference individually, this work considers the characterization of the interference in directional networks jointly with the residual SI from IBFDX systems.

\subsection{Research Question and Motivation}

The key challenge when designing the IBFDX system is to reduce the amount of residual SI. Apart from hardware impairments, the amount the residual SI power is mainly related to the accuracy of the estimated channels. Moreover, directional beamforming plays a major role in boosting the communication quality. In such systems, multiple nodes may act as interference sources toward a specific node. Therefore, it is crucial to study the joint interference caused by not only IBFDX systems, but also by directional beamforming, by answering the following research questions:

- Question 1: What is the best distribution to model the interference power when jointly considering IBFDX and directional communication?

- Question 2: What is the effect of estimation errors on the interference power?

- Question 3: What is the influence of gain pattern parameters (main lobe, sidelobes, and beamwidth) on the interference power?

\section{Relationship to Applied Artificial Intelligence Systems}

Nowadays artificial intelligence (AI) systems play an important role in several sectors, leveraging the added value of a multitude of services through more customized practices that allow a better fit between the service demand and its operational features. Big efforts are usually required to process a large amount of offline data in AI systems, while online applications supported by AI, such as autonomous driving, require very low latency. The low latency requirement includes not only efficient AI algorithms, but also low latency and high throughput communication links. This work is mainly centered on improving the throughput of existing wireless communication systems, which are and will be even more important to deploy a plethora of AI systems capable of addressing mobile and low latency services. 


\section{System Model}

We consider a scenario where nodes are distributed according to a homogeneous Poisson Point Process (PPP) $\Pi$ with density $\lambda$ over an annular region with an inner radius, $R_{\mathrm{I}}$, and an outer radius, $R_{\mathcal{O}}$, i.e., $R_{\mathrm{I}} \leq r \leq R_{\mathcal{O}}$. For the particular case of $R_{\mathrm{I}}=0$ a circular region is considered. Without loss of generality, we condition on a node at the origin, which according to Slivnyak's theory in stochastic geometry [15], yields to a homogeneous PPP with the corresponding density $\lambda$. The reference node is supposed to perform IBFDX to communicate with a corresponding node. At a given time slot, we assume that one or more nodes, except the one connected with the reference node, cause interference. Our goal is to characterize the power of the interference caused by multiple transmitting nodes and the power of the residual SI caused by IBFDX operation.

We consider a full-duplex scheme adopting an active analog canceler [13] that reduces the SI at the angular carrier frequency $\omega_{c}=2 \pi f_{c}$. The model considers the residual interference after the SI signal has been canceled by a generic post-mixer active analog canceller. Analogue cancellation schemes can provide up to 40-50 dB cancellation [10], exhibiting higher performance than digital cancellation. This is explained by the fact that the cancellation signal includes all transmitter impairments. Moreover, an interesting observation reported in [16] is that, if active analog cancellation and digital cancellation are cascaded together, then the amount of digital cancellation depends on the amount of analog cancellation. Consequently, the SI is usually reduced before the digital cancellation through the adoption of analog cancellation techniques. The proposed model considers the residual SI signal in an analog post-mixer canceler, where the canceling signal is generated after the SI signal is upconverted.

The SI signal, $x(t)$, is up-converted to the frequency $\omega_{c}=2 \pi f_{c}$ and transmitted over the full-duplex channel characterized by the gain $h$ and the delay $\tau$. To obtain the residual SI signal, $y_{\text {res }}(t)$, the estimated delay $\hat{\tau}$ and estimated gain $\hat{h}$ have to be injected to perform the cancellation as depicted in Fig. 1.

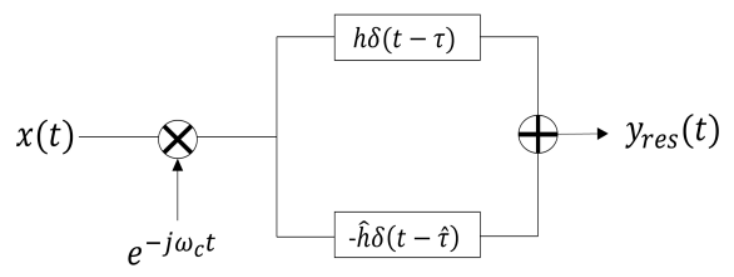

Fig. 1. Block diagram of IBFDX system.

According to Fig. 1, the residual SI sinal, $y_{\text {res }}(t)$, can be expressed as follows

$$
y_{\text {res }}(t)=h x(t-\tau) e^{j \omega_{c}(t-\tau)}-\hat{h} x(t-\hat{\tau}) e^{j \omega_{c}(t-\hat{\tau})},
$$


where $h$ is the channel gain and $\hat{h}$ is the estimated gain given by $\hat{h}=\epsilon h$, where $(1-\epsilon)$ is the gain estimation error, $\epsilon \geq 0$. The phase estimation error is given by $\phi=$ $\omega_{c}(\tau-\hat{\tau})$. The estimation of the parameters $\hat{h}$ and $\hat{\tau}$ can be done using different methods already available in the literature, such as the one described in [17]. We also assume that $x(t)$ is a circularly-symmetric random signal.

Considering that $x(t-\tau)=x(t-\hat{\tau})$, then (1) can be represented as follows

$$
y_{\text {res }}(t)=x(t) h c \text {, }
$$

where $c=\left(e^{j \omega_{c}(t-\tau)}-\epsilon e^{j \omega_{c}(t-\hat{\tau})}\right)$ is a constant. Assuming that $x(t)$ and $h$ are independent random variables (RVs), then, the residual SI power can be expressed as

$$
P_{y_{\text {res }}}=\left(X^{2}\right)\left(H^{2}\right) C
$$

where $C=\left(1+\epsilon^{2}-2 \epsilon \cos (\phi)\right)$ is a constant which represents the power of $c$. According to (3), the residual SI power is proportional to the power of the transmitted signal, the power of the SI channel, and estimation errors.

Considering a large-scale, distance-based path loss and small-scale fading for modeling the wireless channel between any communicating pairs. Therefore, the received power caused by an $i$-th node and seen at the reference node is given by

$$
P_{i}=P_{t} \Psi_{i} G_{i, \jmath} G_{\jmath, i}\left(r_{i}\right)^{-\alpha},
$$

where $P_{t}$ is the same transmitted power across all nodes. We also consider both Rayleigh and Rician small-scale fading channels in which $\Psi_{i}$ is drawn from a Gamma distribution as shown later. $G_{i, \mathrm{I}}$ and $G_{\mathrm{I}, i}$ represent the gain of an $i$-th transmitting node and the gain of the reference node, respectively. $\alpha>2$ is the large-scale path loss coefficient, and $r_{i}$ is the distance between an $i$-th node and the reference node.

The Rayleigh channel can describe the stochastic fading when there is no line-ofsight (LoS) signal. When the channel is a Rayleigh distributed, $\Psi_{i}$ can be drawn from an exponential distribution with mean $1 / \mu$, therefore, it can be expressed by a Gamma distribution with the shape parameter, $k=1$, and the scale parameter, $\theta=\frac{1}{\mu}$, as follows

$$
\Psi_{i} \sim \operatorname{Exp}(\mu) \sim \operatorname{Gamma}(k, \theta) .
$$

On the other hand, the Rician fading channel is parameterized through $K$ and $\Omega$ where $K$ represents the quotient between the power in the LoS component and the power in the other non-LoS components, and $\Omega$ represents the total power from both components. Then, the received signal amplitude is Rician distributed with parameters $v^{2}=\frac{K \Omega}{1+K}$ and $\sigma^{2}=\frac{\Omega}{2(1+K)} . K_{d B}=10 \log _{10}(K)$ is the decibels representation of $K$. By definition, if $X \sim \operatorname{Rice}(v, \sigma)$, then $\left(\frac{\mathrm{X}}{\sigma}\right)^{2}$ follows a non-central Chi-squared distribution with 2 degrees of freedom and non-centrality parameter $\left(\frac{v}{\sigma}\right)^{2}$. Consequently, moment 
matching can then be used to obtain a simplified Gamma approximation for $\Psi_{i}$ as $\Psi_{i} \sim$ $\operatorname{Exp}(\mu) \sim \operatorname{Gamma}(k, \theta)$, where $k$ and $\theta$ are the shape and scale parameters, respectively, given by [18]

$$
k=\frac{\left(v^{2}+2 \sigma^{2}\right)^{2}}{4 \sigma^{2}\left(v^{2}+\sigma^{2}\right)}, \theta=\frac{4 \sigma^{2}\left(v^{2}+\sigma^{2}\right)}{\left(v^{2}+2 \sigma^{2}\right)^{2}} .
$$

Regarding the beamforming model, all nodes are assumed to be equipped with antenna arrays for performing directional beamforming. We adopt a sectorized antenna model to represent the gain patterns $G_{T}$ and $G_{R}$ at the transmitting and receiving node, respectively, as follows

$$
G_{T, R}(\theta)=\left\{\begin{array}{l}
G_{T, R}^{\max },|\theta| \leq \omega / 2 \\
G_{T, R}^{\min },|\theta| \geq \omega / 2
\end{array}\right.
$$

where $G^{\max }$ and $G^{\min }$ are the gains of main and sidelobes, respectively, defined by beamwidth $\omega \in(0,2 \pi)$ and the bore-sight angle direction $\theta \in(-\pi, \pi)$. Without loss of generality, we assume that all nodes are on the same horizontal plane, i.e., no variation in beam pattern over the elevation angle, and we work with the normalized 2-D pattern. While the sectorized antenna modeling is a useful idealization, practical directional antenna gains have a more complex dependence on the azimuth angle. However, useful models with some simplifying assumptions can be obtained and utilized as claimed in [5] and adopted in [13] for establishing innovative MAC policies.

Based on (4), the aggregate interference power caused to the reference node is formulated as

$$
I_{o}=\sum_{i=1}^{N} P_{i}, i \in \Pi,
$$

where $N$ is a RV characterized by a homogeneous PPP that represents the number of active transmitters over the region represented by the area $A=\pi\left(R_{\mathcal{O}}^{2}-R_{\mathrm{I}}^{2}\right)$. Therefore, the probability of $n$ nodes being inside a region $A$ is given by

$$
P[N=n]=\frac{(\lambda|A|)^{n}}{n !} e^{-\lambda|A|} .
$$

Finally, the aggregate interference power at the reference node is the residual SI power given by (3) and the interference power given by (9), which can be written as

$$
P_{\text {tot }}=P_{y_{\text {res }}}+I_{o} \text {. }
$$

\section{Performance Analysis}

The evaluation is based on Monte Carlo simulation results $\left(10^{6}\right.$ realisations of the stochastic process were run). The communication system is operating at a carrier frequency of $60 \mathrm{GHz}$. The residual SI power given in (3) is generated using empirical 
data. The values of $X$ are sampled from Normal distributions, i.e., $X \sim \mathcal{N}\left(0, \sigma_{x}^{2}\right)$ with $\sigma_{x}^{2}=\frac{1}{2}$. The SI channel is assumed to be Rician distributed, i.e., $H \sim \operatorname{Rice}(v, \sigma)$. The aggregate interference power in (8) is also generated using empirical data. $\Psi_{i}$ are generated from a Gamma distribution, where $\mathrm{k}$ and $\theta$ are identified for a Rayleigh channel and for a Rician channel. The network and channel parameters are listed in Table 1, unless otherwise specified.

Table. 1. Network and channel parameters

\begin{tabular}{|c|c|c|c|c|c|c|c|}
\hline$f_{c}$ & $60 \mathrm{GHz}$ & $P_{t}$ & $1 \mathrm{~mW}$ & $\sigma_{x}^{2}$ & 0.5 & $\Omega$ & $1 \mathrm{~mW}$ \\
\hline$\epsilon$ & 0.9 & $\begin{array}{c}K_{d B} \text { for SI } \\
\text { channel }\end{array}$ & 10 & $\sigma_{h}^{2}$ & 0.5 & $\alpha$ & 4 \\
\hline$\phi$ & $10^{\circ}$ & $\begin{array}{c}K_{d B} \text { for } \\
\text { directional } \\
\text { channel }\end{array}$ & 0 & $R_{\mathrm{I}}, R_{\mathcal{O}}$ & $1 \mathrm{~m}, 5 \mathrm{~m}$ & $G^{\text {max }}$ & 1 \\
\hline$\mu$ & 2 & $\omega$ & $\left\{45^{\circ}, 60^{\circ}\right\}$ & $\lambda$ & $\begin{array}{c}50 \text { node } / \\
\mathrm{m}^{2}\end{array}$ & $G^{\text {min }}$ & $\begin{array}{c}\{0.05, \\
0.1\}\end{array}$ \\
\hline
\end{tabular}

The simulated data of the aggregate interference power was used in a maximum loglikelihood estimation process to determine the parameters of the known distributions. The following known distributions were compared: Gamma, Exponential, Nakagami, Rayleigh, Weibull, Generalized Extreme Value (GEV), and Lognormal. Fig. 2 plots the PDF of the distributions that exhibited the best accuracy against the PDF of the empirical data considering a Rician Fading channel for directional communication. As can be seen, GEV distribution exhibits the best accuracy in representing the aggregate interference power. The CDFs of the aggregate interference power are illustrated in Fig. 4 considering Rician and Rayleigh fading channels. Different gain levels of the beam sidelobes and different beamwidths are considered. The results reflect the incremental

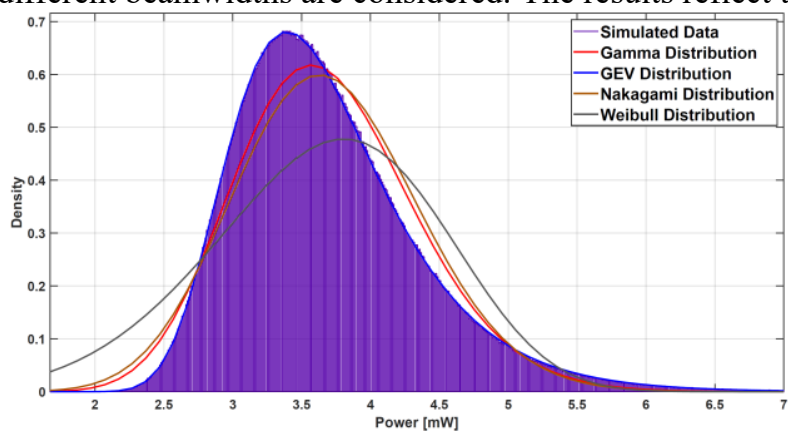

Fig. 2. The PDF of the aggregate interference power with PDFs of other known distributions considering a Rician channel for directional communication: $K_{d B}=0, \omega=45^{\circ}$, and $G^{\text {min }}=0.1$.

effect on the interference power due to the increase in the sidelobes gain $\left(G^{\mathrm{min}}=\right.$ $0.05,0.1$ were adopted in the comparison). On the other hand, the results show that the interference power decreases when adopting small $\omega$ values (narrower beams), due to the concentration of higher power in specific and smaller spatial regions $\left(\omega=45^{\circ}, 60^{\circ}\right.$ were adopted in the comparison). We conclude that although the proposed modeling 
methodology is quite simple, the simulated CDFs comply with different network and channel parameters.
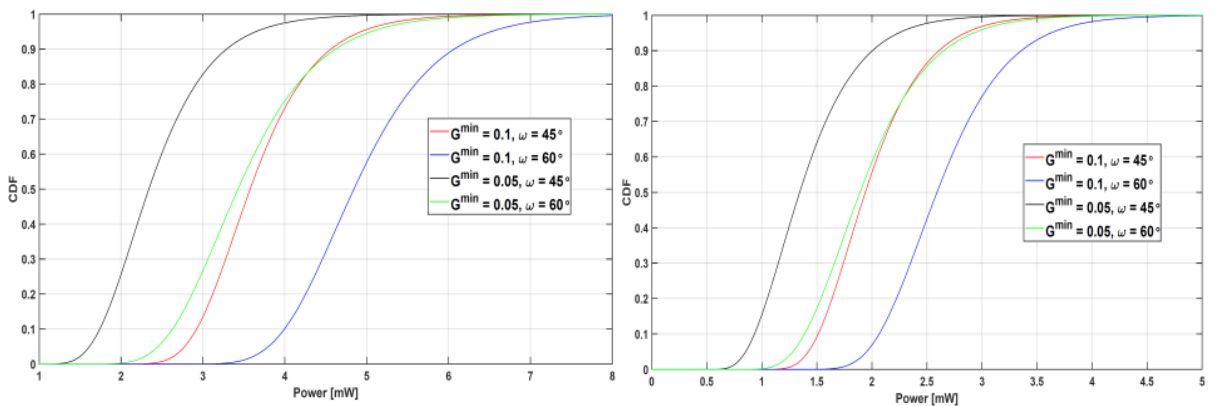

Fig. 4. CDFs of the aggregate interference power $P_{\text {tot }}$ considering 1) a Rician fading channel for directional communication with $K_{d B}=0$ at the left side 2) a Rayleigh fading channel for directional communication: $\mu=2$ at the right side.

\section{Conclusions}

The conclusions of the distribution of the aggregate interference power presented in this paper may be used to provide technical criteria for alleviating the interference in practical directional communication when considering IBFDX systems. One of the practical applications is the computation of the optimal antenna parameters according to the interference level. Moreover, the obtained results can also be helpful for the academic community in general, to determine different aspects related to the performance analysis of both directional communication and IBFDX systems. For example, by using the aggregate interference power to derive the outage probability of a specific system, the capacity of communication systems can be achieved. In this work, we have studied the distribution of the aggregate interference power due to: 1) the residual SI power in IBFDX, 2) the interference caused to a single node in directional beamforming networks. The work takes the initial step for exploring the theoretical distribution of the aggregate interference power. Based on an extensive comparison with known distributions, it is shown that the GEV distribution exhibits the best accuracy in representing the aggregate interference power. Deriving the exact distribution may be considered as future work.

\section{Acknowledgements}

This work was supported by the Marie Skłodowska-Curie project number 813391, TeamUp5G.

\section{References}

1. K. E. Kolodziej, B. T. Perry and J. S. Herd, "In-Band Full-Duplex Technology: Techniques and Systems Survey," in IEEE Trans. on Microw. Theory Tech., vol. 67, no. 7, pp. 30253041, July 2019. 
2. G. C. Alexandropoulos and M. Duarte, "Joint design of multi-tap analog cancellation and digital beamforming for reduced complexity full duplex MIMO systems," IEEE International Conference on Communications (ICC), Paris, 2017, pp. 1-7.

3. H. Yang, M. H. A. J. Herben, I. J. A. G. Akkermans and P. F. M. Smulders, "Impact Analysis of Directional Antennas and Multiantenna Beamformers on Radio Transmission," in IEEE Trans. on Veh. Technol., vol. 57, no. 3, pp. 1695-1707, May 2008.

4. Martin Haenggi and Radha Ganti. Interference in large wireless networks. Foundations and Trends in Networking, 3:127-248, 012009.

5. M. Di Renzo, "Stochastic Geometry Modeling and Analysis of Multi-Tier Millimeter Wave Cellular Networks," in IEEE Trans. on Wireless Comm., vol. 14, no. 9, pp. 5038-5057, Sept. 2015.

6. M. Rebato, J. Park, P. Popovski, E. De Carvalho and M. Zorzi, "Stochastic Geometric Coverage Analysis in mmWave Cellular Networks with Realistic Channel and Antenna Radiation Models," in IEEE Trans. on Commun., vol. 67, no. 5, pp. 3736-3752, May 2019.

7. S. Singh, R. Mudumbai and U. Madhow, "Interference Analysis for Highly Directional 60GHz Mesh Networks: The Case for Rethinking Medium Access Control," in IEEE/ACM Trans. on Netw., vol. 19, no. 5, pp. 1513-1527, Oct. 2011.

8. S. Niknam, B. Natarajan and R. Barazideh, "Interference Analysis for Finite-Area 5G mmWave Networks Considering Blockage Effect," in IEEE Access, vol. 6, pp. 2347023479, 2018

9. H. Elkotby and M. Vu, "Interference Modeling for Cellular Networks Under Beamforming Transmission," in IEEE Trans. on Wireless Commun., vol. 16, no. 8, pp. 5201-5217, Aug. 2017.

10. Sahai, G. Patel, C. Dick, and A. Sabharwal, "On the impact of phase noise on active cancelation in wireless full-duplex," IEEE Trans. Veh. Technol., vol. 62, no. 9, pp. 44944510, Nov 2013.

11. A. T. Abusabah, L. Irio and R. Oliveira, "In-Band Full-duplex Residual Self-interference Approximation in Multi-tap Delay Fading Channels," 2020 International Wireless Communications and Mobile Computing (IWCMC), Limassol, Cyprus, 2020, pp. 635-640.

12. A. T. Abusabah, L. Irio, R. Oliveira and D. B. da Costa, "Approximate Distributions of the Residual Self-Interference Power in Multi-tap Full-Duplex Systems," in IEEE Wireless Commun. Lett., doi: 10.1109/LWC.2020.3042754.

13. L. Irio and R. Oliveira, "Distribution of the Residual Self-Interference Power in In-Band Full-Duplex Wireless Systems," in IEEE Access, vol. 7, pp. 57516-57526, 2019.

14. A. Quazi, "An overview on the time delay estimate in active and passive systems for target localization," in IEEE Trans. Acoust., Speech, Signal Process., vol. 29, no. 3, pp. 527-533, June 1981.

15. Baccelli and B. Blaszczyszyn. Stochastic Geometry and Wireless Networks. Delft, The Netherlands: Now Publishers, Sep.2009.

16. M. Duarte, "Full-duplex wireless: Design, implementation and characterization," Ph.D. dissertation, Rice Univ., Houston, TX, USA, Apr. 2012.

17. Y. Choi and H. Shirani-Mehr, "Simultaneous Transmission and Reception: Algorithm, Design and System Level Performance," in IEEE Trans. on Wireless Commun., vol. 12, no. 12, pp. 5992-6010, December 2013. 